\title{
Proceeding
}

Supplementary Issue: Spring Conferences of Sports Science. Costa Blanca Sports Science Events, 14-15 June 2019. Alicante, Spain.

\section{Effectiveness of multilateral training didactic method on physical and mental wellbeing in law enforcement}

\author{
FRANCESCO FISCHETTI, STEFANIA CATALDI, FRANCESCA LATINO, GIANPIERO GRECO \\ Department of Basic Medical Sciences, Neuroscience and Sense Organs, School of Medicine, University of \\ Study of Bari, Italy
}

\begin{abstract}
Police work requires continuous professional and physical training to cope with high stress situations and meet occupational tasks needs. Therefore, the purpose of this study was to investigate the effects of an 8week multilateral training program on physical and mental wellbeing. 20 healthy male volunteer policemen (age $46.8 \pm 3.9$ years) were randomly assigned to experimental group $(n=10)$ that performed a multilateral training program consisting of agility and resistance training, or control group $(n=10)$. At baseline and after 8 weeks, the sources of stress and coping strategies, and the physical and mental state of health perceived were measured by the Occupational Stress Indicator and the Short Form-12, respectively. The comparison of the mean pre/post differences between the groups was assessed by a two-way repeated measures ANOVA $(p<0.05)$. After multilateral intervention, experimental group perceived less stress, showed a more realistic attitude towards the various working situations and a greater perception of physical and mental wellbeing than control group $(p<0.05)$. The findings suggest that a physical education program in the continuing education framework, through the didactic method of multilateral training, could prevent the occupational stress that causes harmful physical and emotional reactions and threatens the quality of life in police officers. Keywords: Coping strategies; Quality of life; Multilateral didactics; Physical efficiency; Work efficiency.
\end{abstract}

Cite this article as:

Fischetti, F., Cataldi, S., Latino, F., \& Greco, G. (2019). Effectiveness of multilateral training didactic method on physical and mental wellbeing in law enforcement. Journal of Human Sport and Exercise, 14(4proc), S906S915. doi:https://doi.org/10.14198/jhse.2019.14.Proc4.53

Corresponding author. Department of Basic Medical Sciences, Neuroscience and Sense Organs, School of Medicine, University of Study of Bari, Lungomare Starita 1, 70123 Bari (BA). Italy. http://orcid.org/0000-0002-5023-3721

E-mail: gianpiero.greco@uniba.it

Supplementary Issue: Spring Conferences of Sports Science. Costa Blanca Sports Science Events, 14-15 June 2019. Alicante, Spain.

JOURNAL OF HUMAN SPORT \& EXERCISE ISSN 1988-5202

(c) Faculty of Education. University of Alicante

doi:10.14198/jhse.2019.14.Proc4.53

S906 | $2019 \mid$ Proc4 | VOLUME 14

(C) 2019 University of Alicante 


\section{INTRODUCTION}

Police officers experience occupational stress at high levels (Amaranto, Steinberg, Castellano \& Mitchell, 2003; Collins \& Gibbs, 2003; Santana et al., 2012). The National Institute for Occupational Safety and Health defines work-related stress as the body of harmful physical and emotional reactions that occur when job demands are not commensurate with skills, resources or needs (NIOSH, 1999). The main work-related stress in the police recognize different origins: from those linked to the classic "routine work", to organizational, managerial and bureaucratic variables (Kop, Euwena, \& Schaufeli, 1999; Patterson, 2001). It has been shown that high levels of stress in policemen are linked to an increased risk of sub-optimal job satisfaction and quality of life. The size of these associations depends on age, gender and social status, highlighting the need for appropriate training in stress management (Alexopoulos, Palatsidi, Tigani, \& Darviri, 2014).

Perceived stress can contribute to the onset of cardiovascular disease in police officers (Franke, Ramey, \& Shelley, 2002) and routine work can be significantly more stressful than a single critical event (Garbarino et al., 2011). In fact, the levels of work-related stress are significantly higher during routine public order activities, such as migration control activities, than during extraordinary public order activities, probably due to positive changes in the psychosocial components of the working environment (Garbarino, Magnavita, Cipriani, \& Cuomo, 2011). Work-related stress is associated with a lower level of psychological wellbeing and an increase in the frequency of absences from work (Magnavita, Garbarino, \& Siegrest, 2014). To this end, to improve work performance, it has been suggested to include some psychological pressure in the simulations during training (Nieuwenhuys, Caljouw, Leijsen, Schmeits, \& Oudejans, 2009). The most appropriate strategies used by law enforcement to modulate stress and reduce it are based on achieving greater personal self-esteem that leads to positive effects on mental health and wellbeing, and to optimal interpretation of events (Gana \& Boblique, 2000). Furthermore, communication in interpersonal and family relations is also an important strategy (Burke, 1993). Optimum use of leisure time (sports activities, friendships, etc.) is also suggested for the ability to improve mood, facilitating a momentary coping of tension situations where engagement in positive support groups outside the workplace can help to improve stress levels (Fischetti \& Greco, 2017c; Greco \& Fischetti, 2018; Iwasaki, Mannell, Smale, \& Butcher, 2005).

A careful analysis of the literature has shown that law enforcement is often exposed to periods of intense work stress to ensure investigation, surveillance and public order. Thus, the development of a physical education didactic method is necessary to ensure that officers are able to efficiently perform police work (Bonneau \& Brown, 1995; Trottier \& Brown, 1994). With regard to previous findings, we wanted to focus this research on a didactic method of multilateral training including primarily muscular strength and agility, which are regarded as two components of physical fitness. Resistance training (i.e., muscular strength training) is effective in enhancing several important aspects of physical and mental health. In fact, it may decrease symptoms of depression, increase self-esteem and physical self-concept, and improve cognitive ability (Westcott, 2012). In addition, agility training appears to be effective in enhancing specific measures of cognitive performance, such as memory, and vigilance (Lennemann et al., 2013).

According to these considerations, it is reasonable to assume that interventional studies are necessary to ensure the physical and mental well-being of officers and improve their quality of life, because this occupation requires the maintenance of adequate physical and mental health condition (Da Silva et al., 2014). Therefore, the purpose of this study was to investigate the effects of an 8-week multilateral training program on physical and mental wellbeing in policemen. We hypothesized that the ways of coping with stress and the job stressors perception would improve as well as the physical and mental health perception after intervention. 


\section{MATERIALS \& METHODS}

\section{Study design}

This research was developed through a randomized controlled study design in order to collect the data from standardized psychological instruments (i.e., Occupational Stress Indicator and Short Form-12 health survey) and compare the two group at different times with respect to the measured dependent variables. Data were collected and recorded at baseline (Pre-test) and after 8 weeks (Post-test).

\section{Participants}

A sample of 20 healthy males (age $46.8 \pm 3.9$ years; stature $173.8 \pm 6.6 \mathrm{~cm}$; body weight $81.2 \pm 14.1 \mathrm{~kg}$, mean \pm SD) belonging to the State Police voluntarily participated in the study. Participants were recruited in the police offices of the Puglia region (Italy) between January and February 2019. An a priori power analysis (Faul, Erdfelder, Lang \& Buchner, 2007) with an assumed Type I error of 0.05 and a Type II error rate of 0.20 ( $80 \%$ statistical power) was calculated and revealed that eight participants per group would be sufficient to observe medium "Time x Group" interaction effects. The anticipated number of participants was increased to prevent expected drop out. All participants were eligible for inclusion in this study because they had no history of musculoskeletal, neurological, or orthopedic disorders that might have affected their ability to perform the physical training program. All of the measurement and training procedures and potential risks were verbally explained to each participant, and their informed consent was obtained. After testing, participants were randomly assigned to experimental group $(n=10)$ that performed a multilateral training program consisting of agility and resistance training, or control group $(n=10)$. The control group will carry out the same intervention program at a later date. The study was carried out between the months of March and April 2019. All participants completed the study. The study procedures were followed in accordance with the ethical standards of the local responsible institutional committee on human experimentation and with the Helsinki Declaration.

\section{Procedures and measures}

In this study, self-administered paper-based questionnaires were used, to be completed anonymously, after the prior consent of the State Police Personnel Manager. The questionnaires were administered under the same conditions, with a setting free of distractions and in comfortable space. It has been explained to compile in a systematic, fast and accurate way, but without time limits.

To assess psychosocial stress and physical and mental health were administered the versions translated and validated in Italian of: a) two sections of the Occupational Stress Indicator (Cooper, Sloan \& Williams, 1988): "Sources of stress" e "Coping strategies"; b) the Short Form-12 health survey (Ware, Kosinsky \& Keller, 1996; Apolone et al., 2001) which can be considered an indicator of the physical and mental health.

The Occupational Stress Indicator (OSI) is considered a useful indicator for detection of psychosocial stress in organizations. The "job stressors" section consists of 61 six-point Likert scale items and includes six subscales: (1) Intrinsic factors to the job: stress is perceived on the basis of how the group or individual engages their working days; (2) Management role: stress comes from living according to the expectations of one's own role; (3) Relationship with others: interpersonal stress is perceived; (4) Career and achievement: stress associated with personal develop; (5) Organizational structure and climate: stress is the result of a feeling of frustration due to the characteristics of the organization; (6) Home/work interface: stress depends on the characteristics of the family environment or an intrusion of work into family life. A high score means that a source of pressure is relevant for the specific function in question. In this section, the Cronbach's alpha coefficient showed an excellent level of reliability and internal consistency for its subscales $(\alpha=0.90-0.96)$. 
The "ways of coping with stress" section consists of 28 six-point Likert scale items and includes six subscales: (1) Social support: the way people rely on others as a means of coping with stress; (2) Task strategies: the way people cope with stress by reorganizing work; (3) Logic: the way to cope with stress by adopting a rational and not an emotional approach to the situation; (4) Home/work relations: concerns the use of resources outside work to reintegrate one's own capacity to cope with stress; (5) Time management: relates to time management; (6) Involvement: refers to having a realistic picture of what is possible. High scores suggest that the strategy is used more often. In this section, the Cronbach's alpha coefficient showed a good level of reliability and internal consistency for its subscales $(\alpha=0,82-0.89)$.

The Short Form-12 (SF-12) is a questionnaire that describes the physical and mental health of a group of people using 12 Likert scale items consisting of two questions for each of the SF-36 scales (Apolone \& Mosconi, 1998). The synthetic indexes of the SF-12 questionnaire are the PCS-12 (Physical Component Summary) and MCS-12 (Mental Component Summary): PCS reflects concepts related to physical morbidity, while MCS reflects morbidity and psychological and mental aetiologies. The scores range from 0 (worst health condition) to 100 (best health condition). Low PCS scores indicate substantial limitations in self-care and physical, social and personal activity; severe physical pain; frequent fatigue; health is judged to be poor. High scores in PCS indicate no physical limitation, disability or decrease in general well-being; high vitality; health is considered excellent. Low scores in MCS indicate frequent psychological discomfort; important social and personal disability due to emotional problems; health is considered poor. High scores in MCS indicate frequent positive psychological attitude; absence of psychological discomfort and limitations in social and personal activities due to emotional problems; health is considered excellent. The reliability and internal consistency level for SF-12 was excellent, determined by a Cronbach's alpha of 0.92 .

\section{Multilateral training program}

The subjects allocated to the experimental group received a multilateral training program for a period of 90 minutes, two days a week, with a total of 16 training sessions. These training sessions were performed at different times of the day, depending on the availability of the policemen outside working hours. The entire intervention program was performed over eight weeks and supervised and conducted by two experienced instructors, who are graduates in physical education. The participants were instructed to report injuries sustained during training. Each training session started with a brief dynamic warm-up program, mainly consisting of calisthenics-type exercises for approximately ten minutes, and ended with a cool-down program, consisting of static stretching exercises for approximately ten minutes. The targeted components of the multilateral training program mainly included lower limb strength training and agility in addition to training for the upper limbs to enhance a balanced physique. On the first day, the training was focused on plyometrics and weight training. On the second day, plyometrics and agility training were performed. During the first two weeks, the training primarily consisted of preconditioning. From the third week onwards, we gradually increased the intensity and volume of the training. For an effective workout, the principles of overload and specificity were addressed (Baechle \& Earle, 2008).

The dynamic warm-up program included arm swings, trunk twisting, high marching, jumping jacks, stride jumping, high knees, side bending, side stretching, skipping leg swings, backward sprinting, carioca, and lateral shuffles. The subjects performed each exercise for $1 \mathrm{set}$ of $60 \mathrm{~s}$.

The resistance training protocol included plyometrics and weight training. During body weight plyometrics, the subjects performed Jump Squats, burpees, lunges, push-ups, pull-ups, sit-ups/crunches, back extensions, single or double leg hops, half squats, long jumps, planks, and medicine ball tosses. In total, 1 2 sets of 10 reps, with $30-60 \mathrm{~s}$ of slow walking between each exercise, were performed. During weight 
training, the subjects performed $2-3$ sets of $8-12$ reps ( $60 / 80 \%$ of $1 \mathrm{RM})$, with 1 -minute rest periods in between sets. The 10 exercises performed included leg presses, leg extensions, leg curls, lat pulldowns, seated rows, bench presses, shoulder presses, triceps presses, arm curls, and calf raises.

The aims of the agility training protocol were to challenge hand-eye coordination, foot-eye coordination, dynamic balance, standing and leaning balance, and psychomotor performance (reaction time). Ball games, relay races, box drills, shuttle run, lateral coach drills, and obstacle courses were used to achieve these goals. The agility training was performed for 30-40 minutes.

The cool-down program was performed with static stretches, which included movements, such as Achilles' tendon/calf stretches, skier's stretches, quadriceps stretches, hurdler's stretches, straddle stretches, groin stretches, back stretches, and archers.

\section{Statistical analyses}

All analyses were performed using SAS JMP® Statistics (Version <14.1>, SAS Institute Inc., Cary, NC, USA, 2018) and the data are presented as group mean values and standard deviations. Normality of all variables was tested using Shapiro-Wilk test procedure. A multivariate analysis of variance (MANOVA) was used to detect differences between the study groups in all baseline variables. Training-related effects were assessed by 2-way analyses of variance (ANOVA) with repeated measures (group $x$ time). When 'Time $x$ Group' interactions reached the level of significance, group-specific post hoc tests (i.e., paired t-tests) were conducted to identify the significant comparisons. Cohen's $d$ effect size was calculated as post-training mean minus pre-training mean divided by pooled SD before and after training, and interpreted as small, moderate and large effects defined as $0.20,0.50$, and 0.80 , respectively (Cohen, 1992). The reliabilities of the psychological measures were assessed using Cronbach's alpha coefficient and intraclass correlation coefficients, respectively; scores from 0.8 to 0.9 were considered as high reliable, while values above $>0.9$ were considered as very high reliable (Cohen, Manion, \& Morrison, 2011). We accepted $p \leq 0.05$ as our criterion of statistical significance, whether a positive or a negative difference was seen (i.e., a 2-tailed test was adopted).

\section{RESULTS}

All participants received the treatment conditions as allocated and the adherence to training was $100 \%$. All participants completed the multilateral intervention and no major adverse effect and no major health problem were noted in the participants over the 8-week period. Both groups did not differ significantly at baseline in age, anthropometric characteristics, as well as in psychological measures $(p>0.05)$. Pre- and postintervention results for all outcome measures are presented in Table 1.

\section{OSI- Sources of stress}

Group by time interactions were observed for all subscales $(p<0.001)$. After 8 weeks, the experimental group showed decreased scores than control group with statistically significant within-group changes for: "Intrinsic factors to the job" (FJ: $p<0.001$ ), "Management role" (FM: $p<0.001$ ), "Relationship with others" (FR: $p<$ 0.01 ), "Career and achievement" (FC: $p<0.001$ ), "Organizational structure and climate" (FS: $p<0.001)$, and "Home/work interface" (Fl: $p<0.001)$. Changes meaningful corresponded moderate to large effect sizes $(d$ $=0.72-1.26$.) 
Table 1. Changes in psychological measures after 8-week multilateral training intervention

\section{Experimental group \\ Control group}

$(n=10)$

$(n=10)$

\begin{tabular}{lcccccc}
\hline Variables & Pre-test & Post-test & $\Delta$ & Pre-test & Post-test & $\Delta$ \\
\hline Occupational Stress Indicator & & & & & & \\
Sources of stress & & & & & & \\
Job (FJ) & $30.7(5.2)$ & $25.2(5.3) \dagger^{*}$ & -5.5 & $30.5(4.9)$ & $30.4(5.2)$ & -0.1 \\
Management role (FM) & $39.5(9.0)$ & $35.1(9.9) \dagger^{*}$ & -4.4 & $38.2(8.4)$ & $37.8(8.2)$ & -0.4 \\
Relationship (FR) & $33.2(8.9)$ & $28.3(8.9) \dagger^{*}$ & -4.9 & $33.2(7.5)$ & $33.0(7.3)$ & -0.2 \\
Career and achievement (FC) & $32.1(6.5)$ & $27.5(7.1) \dagger^{*}$ & -4.6 & $32.6(5.9)$ & $33.4(5.6)^{*}$ & 0.8 \\
Organizational climate (FS) & $38.6(8.5)$ & $34.3(9.7) \dagger^{*}$ & -4.3 & $38.0(8.7)$ & $38.1(8.5)$ & 0.1 \\
Home/work (FI) & $38.6(6.9)$ & $33.8(7.6) \dagger^{*}$ & -4.8 & $38.2(5.0)$ & $39.2(5.1)^{*}$ & 1.0 \\
Coping strategies & & & & & & \\
Social support (CS) & $18.5(3.6)$ & $21.0(3.3) \dagger^{*}$ & 2.5 & $18.9(3.4)$ & $19.1(3.2)$ & 0.2 \\
Task strategies (CP) & $28.8(7.3)$ & $32.3(7.3) \dagger^{*}$ & 3.5 & $28.3(5.9)$ & $27.8(6.1)$ & -0.5 \\
Logic (CL) & $13.5(2.5)$ & $13.9(2.8)$ & 0.4 & $13.8(2.7)$ & $14.1(2.0)$ & 0.3 \\
Home/work relations (CR) & $19.2(2.7)$ & $22.7(2.3) \dagger^{*}$ & 3.5 & $18.3(3.6)$ & $18.3(3.1)$ & 0.0 \\
Time management (CT) & $18.9(2.1)$ & $16.3(1.9) \dagger^{*}$ & -2.6 & $17.4(2.0)$ & $17.7(2.3)$ & 0.3 \\
Involvement (CI) & $30.2(3.8)$ & $32.7(3.2) \dagger^{*}$ & 2.5 & $29.9(3.1)$ & $29.2(3.1)$ & -0.7 \\
Short Form-12 & & & & & & \\
PCS-12 & $47.7(2.7)$ & $52.4(2.1) \dagger^{*}$ & 4.7 & $48.5(3.5)$ & $48.4(3.9)$ & -0.1 \\
MCS-12 & $48.2(4.9)$ & $53.4(4.4) \dagger^{*}$ & 5.2 & $48.5(3.5)$ & $44.3(13.6)$ & -1.2 \\
\hline Not: valus ar prosen
\end{tabular}

Note: values are presented as mean ( \pm SD); $\triangle$ : pre-post mean difference; PCS: Physical Component Summary; MCS: Mental Component Summary. *Significantly different from pre-test $(p<0.01)$. S Significant 'Time $x$ Group' interaction = significant effect of the multilateral intervention $(p<0.01)$.

\section{OSI- Coping strategies}

Group by time interactions were observed for all subscales $(p<0.01)$ except for "Logic" (CL: $p=0.85)$. After intervention, the experimental group showed increased scores than control group with statistically significant within-group changes for: "Social support" (CS: $p<0.001$ ), "Task strategies" (CP: $p<0.001$ ), "Home/work relations" (CR: $p<0.001)$ and "Involvement" ( $\mathrm{Cl}: p<0.001)$. Instead, significant decreased scores were detected in the subscale "Time management" (CT: $p<0.001)$ for experimental group. Changes meaningful corresponded moderate to large effect sizes $(d=0.64-0.86)$.

\section{SF-12}

Group by time interactions were observed for the synthetic indexes of the SF-12 questionnaire $(p<0.001)$. After intervention, the experimental group showed increased scores than control group with significant changes from pre- to post-intervention for PCS-12 ( $p<0.001, d=0.89)$ and MCS-12 $(p=0.035, d=0.74)$.

\section{DISCUSSION}

This study aimed to investigate the stress management and the perception of the physical and mental health of the policemen after an 8-week multilateral training intervention (i.e., muscular strength and agility training). 
It has been found that police officers perceived fewer sources of pressure after intervention and specifically: working days are lived with less stress; living the role itself does not create particular conflicting expectations; a particular interpersonal stress is not perceived; particular frustrations linked to personal develop or due to the characteristics of the organization are not experienced; finally, the family environment can contribute more to the acquisition of new resources than the representative population. To cope with stress, police officers seem to rely more on others, reorganize their work more often, use resources outside of work more (e.g. sports activities in leisure time), show more efficient control of their time probably defending it from the demands of others and, finally, present a more realistic attitude with greater self-commitment in front of the various working situations. In addition, police officers deal with situations as rationally as before multilateral intervention. Finally, they showed a greater perception of physical and mental well-being after intervention.

In this study, the results showed that police officers have used appropriate coping strategies and had a greater resilience to stress after physical education program (Bonneau \& Brown, 1995; Trottier \& Brown, 1994). Furthermore, the multilateral approach (i.e., resistance and agility training) was effective to improve physical and psychology fitness (Da Silva et al., 2014; Fischetti, Cataldi, Di Terlizzi, \& Greco, 2019; Greco \& Fischetti, 2018; Lennemann et al., 2013; Magnavita et al., 2014; Westcott, 2012). Thus, the multilateral training didactic method provided the policemen a possibility to use positive coping strategies to address events (Acquadro Maran et al., 2015; Gana \& Boblique, 2000) by placing emphasis on interpersonal and family relationships in line with the assertion of Burke (1993) and by appropriately using free time to find resources outside work (i.e., physical and sports activity) to reduce stress, in accordance with the study of Iwasaki et al. (2005).

Although the stressful nature of police work can cause musculoskeletal disorders (Beyaz \& Ketenci, 2010), the perception of the physical wellbeing of the police officers was higher after intervention (Brown, Wells, Trottier, Bonneau, Ferris, 1998). In addition, the results showed that the police officers have reached a better mental health status (Van der Velden et al., 2013). This could be since trained policemen could use appropriate stress management strategies to control chronic fatigue at work and plan private life (Deschamps, Paganon-Badinier, Marchand, \& Merle, 2003; Stepka \& Basinska, 2014). Thus, based on previous research (Acquadro Maran et al., 2015; Alexopoulos et al., 2014; Fischetti \& Greco, 2017a, 2017b, 2017c; Fischetti et al., 2019; Greco \& Fischetti, 2018; Lagestad, Jenssen, \& Dillern, 2014; Magnavita et al., 2014; Nieuwenhuys et al., 2009; Norvell \& Belles, 1993) and results observed in this study, we can state that a physical education program with multilateral approach could play an important role in providing strategies to prevent and manage stress and maintain a state of physical and mental well-being, fundamental conditions to improve the quality of life and physical and working efficiency of police officers.

This research has limitations that must be known. First, the small number of police officers recruited due to the difficulties encountered during the organizational phase in obtaining the necessary authorizations and having the subjects available. Moreover, the voluntary sample is not representative of the entire population of the law enforcement and therefore it is not possible to generalize the results. However, the results obtained could provide important indications for future studies aimed to know the effects of physical training with a multilateral approach on the occupational stress management.

\section{CONCLUSIONS}

In summary, this study contains some strengths, it provides important evidence in the prevention of workrelated stress in the law enforcement. A Physical Education Program in Continuing Education designed for the police officers is strongly recommended. In policing, the didactic method of multilateral training is effective 
in increasing physical and psychological fitness and improving quality of life. Training should continue beyond eight weeks and should be targeted toward job specific training tasks. A physical education graduate instructor is necessary to design and administer a periodized training program for this population. Consequently, public policies are needed to promote the practice of physical activities as continuing education, including leisure and sports activities, and to promote the psychological stability, work efficiency, changes in living habits, improvements in wellbeing and, therefore, improvements in quality of life.

\section{FUNDING}

No sources of funding were used to assist in the preparation of this manuscript.

\section{CONFLICT OF INTEREST STATEMENT}

The authors declared no potential conflicts of interest with respect to the research, authorship, and/or publication of this article.

\section{AUTHORS' CONTRIBUTION}

FF designed the study, interpreted the data, wrote and revised the manuscript. SC collected and interpreted the data and wrote the manuscript. FL collected and interpreted the data. GG designed the study, carried out the statistical analysis, interpreted the data, wrote and revised the manuscript. All authors contributed intellectually to the manuscript, and all authors have read the manuscript and approved the submission.

\section{REFERENCES}

Acquadro Maran, D., Varetto, A., Zedda, M., \& leraci, V. (2015). Occupational stress, anxiety and coping strategies in police officers. Occupational Medicine, 65(6), 466-73. https://doi.org/10.1093/occmed/kgv060

Alexopoulos, E. C., Palatsidi, V., Tigani. X., \& Darviri, C. (2014). Exploring stress levels, job satisfaction, and quality of life in a sample of police officers in Greece. Safety and Health at Work, 5(4), 210-215. https://doi.org/10.1016/.shaw.2014.07.004

Amaranto, E., Steinberg, J., Castellano, C., \& Mitchell, R. (2003). Police stress interventions. Brief Treatment and Crisis Intervention, 3, 47-53. https://doi.org/10.1093/brief-treatment/mhg001

Apolone, G., \& Mosconi P. (1998). The Italian SF-36 Health Survey: translation, validation and norming. Journal of Clinical Epidemiology, 51, 1025-1036. https://doi.org/10.1016/s0895-4356(98)00094-8

Apolone, G., Mosconi, P., Quattrociocchi, L., Gianicolo, E., Groth, N. \& Ware Jr, J. E. (2001). Questionario sullo stato di salute SF-12 [Health Status Questionnaire SF-12]. Milano, Italia: Istituto di Ricerche farmacologiche Mario Negri.

Baechle, T.R., \& Earle, R.W. (2008). Essentials of strength training and conditioning. Champaign, IL: Human Kinetics.

Beyaz, E. A., \& Ketenci, A. (2010). Low back pain in policemen. Agri, 22(1), 1-6.

Bonneau, J., \& Brown, J. (1995). Physical ability, fitness and police work. Journal of Clinical Forensic Medicine, 2, 157-164. https://doi.org/10.1016/1353-1131(95)90085-3

Brown, J. J., Wells, G. A., Trottier, A. J., Bonneau, \& J., Ferris, B. (1998). Back pain in a large Canadian police force. Spine (Phila Pa 1976), 23(7), 821-827. https://doi.org/10.1097/00007632-199804010$\underline{00017}$ 
Burke, R. J. (1993). Work-family stress, conflict, coping and burnout in police officers. Stress Medicine, 9, 171-180. https://doi.org/10.1002/smi.2460090308

Cohen, J. (1992). A Power Primer. Psychological Bulletin, 112(1), 155-159.

Cohen, L., Manion, L., \& Morrison, K. (2011). Research Methods in Education (7th edition). Oxford, UK: Routledge.

Collins, P. A., \& Gibbs, A. C. (2003). Stress in police officers: a study of the origins, prevalence and severity of stress-related symptoms within a county police force. Occupational Medicine, 53(4), 256264. https://doi.org/10.1093/occmed/kgg061

Cooper, C. L., Sloan, S. J., \& Williams, S. (1988). Occupational Stress Indicator: Management guide. Windsor, UK: NFER-Nelson (Italian translation by S. Sirigatti \& C. Stefanile. Firenze, Italia: Giunti O.S. Organizzazioni Speciali, 2002).

Da Silva, F.C., Hernandez, S.S., Goncalves, E., Arancibia, B.A., Da Silva Castro, T.L., \& Da Silva, R. (2014). Anthropometric indicators of obesity in policemen: a systematic review of observational studies. International Journal of Occupational Medicine and Environmental Health, 27, 891-901. https://doi.org/10.2478/s13382-014-0318-0

Deschamps, F., Paganon-Badinier, I., Marchand, A. C., \& Merle, C. (2003). Sources and assessment of occupational stress in the police. Journal of Occupational Health, 45(6), 358-364. https://doi.org/10.1539/joh.45.358

Faul, F., Erdfelder, E., Lang, A.G., \& Buchner, A. (2007). G*Power 3: a flexible statistical power analysis program for the social, behavioral, and biomedical sciences. Behavior Research Methods, 39, 175191. https://doi.org/10.3758/bf03193146

Fischetti, F., Cataldi, S., Di Terlizzi, P.G., \& Greco, G. (2019). Multilateral methodology in physical education improves coping skills, resilience and physical fitness in drug addicts. Journal of Human Sport and Exercise. https://doi.org/10.14198//hse.2020.152.11

Fischetti, F., \& Greco, G. (2017a). Long-term motor and sports educational practice and active lifestyle: prevention of occupational stress in Police and psychophysical well-being. Formazione \& Insegnamento, 15(Suppl 2), 129-140.

Fischetti, F., \& Greco, G. (2017b). Multilateral methods in Physical Education improve physical capacity and motor skills performance of the youth. Journal of Physical Education and Sport, 17(Suppl 4), 2161-2168. https://doi.org/10.7752/jpes.2017.s4223

Fischetti, F., \& Greco, G. (2017c). Multilateral training as an innovative didactic method to support continuous education in police. Form@re, 17(3), 303-315. http://dx.doi.org/10.13128/formare-20801

Franke, W. D., Ramey, S. L., \& Shelley 2nd, M. C. (2002). Relationship between cardiovascular disease morbidity, risk factors, and stress in a law enforcement cohort. Journal of Occupational and Environmental Medicine, 44(12), 1182-1189. https://doi.org/10.1097/00043764-200212000-00014

Gana, K., \& Boblique, C. (2000). Coping and burnout among police officers and teachers: Test of a model. European Review of Applied Psychology/Revue Europeenne de Psychologie Appliquee, 50(4), 423-430. https://doi.org/10.1016/i.erap.2003.12.003

Garbarino, S., Magnavita, N., Ciprani, F., \& Cuomo, G. (2011). The coping and support activities in the phenomenon of mass immigration in Italy: evaluation of work-related stress in police forces. Giornale Italiano di Medicina del Lavoro ed Ergonomia, 33(3), 170-174.

Garbarino, S., Magnavita, N., Elovainio, M., Heponiemi, T., Ciprani, F., Cuomo, G., \& Bergamaschi, A. (2011). Police job strain during routine activities and a major event. Occupational Medicine, 61(6), 395-399. https://doi.org/10.1093/occmed/kgr058

Greco, G., \& Fischetti, F. (2018). Physical, technical and tactical training and stress management in law enforcement. Journal of Physical Education and Sport, 18(2), 555-560. 
Iwasaki, Y., Mannell, R. C., Smale, B. J., \& Butcher, J. (2005). Contributions of leisure participation in predicting stress coping and health among police and emergency response services workers. Journal of Health Psychology, 10(1), 79-99. https://doi.org/10.1177/1359105305048557

Kop, N., Euwena, M., \& Schaufeli, W. (1999). Burnout, job stress and violent behaviour among Dutch police officers. Work \& Stress, 13(4), 326-340. https://doi.org/10.1080/02678379950019789

Lagestad, P., Jenssen, O. R., \& Dillern, T. (2014). Changes in Police Officers' Physical Performance after 16 Years of Work. International Journal of Police Science \& Management, 16(4), 308-317. https://doi.org/10.1350/ijps.2014.16.4.349

Lennemann, L.M., Sidrow, K.M., Johnson, E.M., Harrison, C.R., Vojta, C.N., \& Walker, T.B. (2013). The influence of agility training on physiological and cognitive performance. The Journal of Strength \& Conditioning Research, 27, 3300-3309. https://doi.org/10.1519/jsc.0b013e31828ddf06

Magnavita, N., Garbarino, S., \& Siegrist, J. (2014). Metodi di valutazione dello stress lavoro-correlato nelle Forze dell'Ordine [Methods of assessing work-related stress in law enforcement]. Giornale Italiano di Medicina del Lavoro ed Ergonomia, 36(4), 400-404. https://doi.org/10.1007/s13631-017$\underline{0149-7}$

Nieuwenhuys, A., Caljouw, S. R., Leijsen, M. R., Schmeits, B. A., \& Oudejans, R. R. (2009). Quantifying police officers' arrest and self-defence skills: does performance decrease under pressure? Ergonomics, 52(12), 1460-1468. https://doi.org/10.1080/00140130903287981

Norvell, N., \& Belles, D. (1993). Psychological and physical benefits of circuit weight training in law enforcement personnel. Journal of Consulting and Clinical Psychology, 61(3), 520-527. https://doi.org/10.1037//0022-006x.61.3.520

Patterson, G. T. (2001). The relationship between demographic variables and exposure to traumatic incidents among police officers. The Australian Journal of Disaster and Trauma Studies, 2001-2.

Santana, A. M., Gomes, J. K., De Marchi, D., Girondoli, Y. M., Rosado, L. E., Rosado, G. P., \& de Andrade, I. M. (2012). Occupational stress, working condition and nutritional status of military police officers. Work, 41(1), 2908-2914.

Stepka, E., \& Basińska, M. A. (2014). Chronic fatigue and strategies of coping with occupational stress in police officers. Medycyna Pracy, 65(2), 229-238. https://doi.org/10.13075/mp.5893.2014.033

The National Institute for Occupational Safety and Health (1999). Stress at work. (Publication No. 99101). Cincinnati, $\mathrm{OH}$ : DHHS(NIOSH).

Trottier, A., \& Brown, J. (1994). Police health: a physician's guide for the assessment of police officers. Ottawa: Canadian Communication Group - Publishing.

Van der Velden, P. G., Rademaker, A. R., Vermetten, E., Portengen, M. A., Yzermans, J. C., \& Grievink, L. (2013). Police officers: a high-risk group for the development of mental health disturbances? A cohort study. BMJ Open, 3(1). https://doi.org/10.1136/bmjopen-2012-001720

Ware, J., Kosinsky, M., \& Keller, S.D. (1996). 12-Item Short-Form Health: construction of scales and preliminary tests of reliability and validity. Medical Care, 34, 220-233. https://doi.org/10.1097/00005650-199603000-00003

Westcott, W.L. (2012). Resistance training is medicine: effects of strength training on health. Current Sports Medicine Reports, 11, 209-216. https://doi.org/10.1249/jsr.0b013e31825dabb8

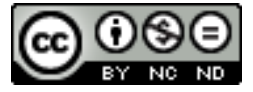

This work is licensed under a Attribution-NonCommercial-NoDerivatives 4.0 International (CC BY-NC-ND 4.0). 